\title{
Erratum to: Temperature-induced variation in gene expression burst size in metazoan cells
}

Ophélie Arnaud ${ }^{1,5}$, Sam Meyer ${ }^{2,6}$, Elodie Vallin ${ }^{1,7}$, Guillaume Beslon ${ }^{2,4}$ and Olivier Gandrillon ${ }^{1,3,7^{*}}$

\section{Erratum to: BMC Molecular Biol (2015) 16:20 DOI 10.1186/s12867-015-0048-2}

Unfortunately, the original version of this article [1] contained two mistakes. The figure order was incorrectly captured during the production process with Figure 1 intended as Figure 6. All other figures are renumbered to accommodate this. The reference to the incorrect Figure 1 in the text should also have read, "(2) the RNA half-life, and it is further validated by the good agreement of the model with experimental curves (see below)."

The original version of the article has been updated to rectify these errors.

\section{Author details}

${ }^{1}$ Centre de Génétique et de Physiologie Moléculaire et Cellulaire (CGPhiMC), CNRS UMR5534, Université de Lyon, Université Lyon 1, 69622 Lyon, France.

${ }^{2}$ Laboratoire d'InfoRmatique en Image et Systèmes d'information (LIRIS), CNRS UMR5205, INSA-Lyon, INRIA, Université de Lyon, 69621 Lyon, France. ${ }^{3}$ Inria Team Dracula, Inria Center Grenoble Rhône-Alpes, Montbonnot-Saint-Martin, France. ${ }^{4}$ Inria Team Beagle, Inria Center Grenoble Rhône-Alpes, Montbonnot-Saint-Martin, France. ${ }^{5}$ Present Address: Division of Genomic Technologies, RIKEN Center for Life Science Technologies, Yokohama, Japan. ${ }^{6}$ Present Address: INSA-Lyon, CNRS UMR5240 Microbiologie, Adaptation et Pathogénie, Université de Lyon, 69622 Lyon, France. ${ }^{7}$ Present Address: Laboratoire de Biologie Moléculaire de la Cellule, Ecole Normale Supérieure de Lyon, CNRS, Université de Lyon, 46 Allée d'Italie, 69007 Lyon, France.
The online version of the original article can be found under doi:10.1186/s12867-015-0048-2.

Received: 28 December 2015 Accepted: 29 December 2015 Published online: 03 February 2016

\section{Reference}

1. Arnaud O, Meyer S, Vallin E, Beslon G, Gandrillon O. Temperature-induced variation in gene expression burst size in metazoan cells. BMC Mol Biol. 2015;16:20. doi:10.1186/s12867-015-0048-2.

\footnotetext{
*Correspondence: Olivier.Gandrillon@univ-lyon1.fr; olivier.gandrillon@ ens-lyon.fr

${ }^{7}$ Present Address: Laboratoire de Biologie Moléculaire de la Cellule, Ecole Normale Supérieure de Lyon, CNRS, Université de Lyon, 46 Allée d'Italie, 69007 Lyon, France

Full list of author information is available at the end of the article
} 